Revue scientifique sur la conception et l'aménagement de l'espace

\title{
Les jardins flottants du lac Inlé (Birmanie), ou la construction d'un paysage agricole et touristique original
}

The Floating Gardens of Lake Inle (Burma), Or the Building of an Original Agricultural and Touristic Landscape

\section{Martin Michalon}

\section{OpenEdition}

\section{Journals}

Édition électronique

URL : http://journals.openedition.org/paysage/8291

DOI : 10.4000/paysage.8291

ISSN : 1969-6124

Éditeur :

École nationale supérieure du paysage de Versailles-Marseille, Institut national des sciences appliquées Centre Val de Loire - École de la nature et du paysage, École nationale supérieure d'architecture et de paysage de Bordeaux, École nationale supérieure d'architecture et de paysage de Lille, Agrocampus Angers

\section{Référence électronique}

Martin Michalon, « Les jardins flottants du lac Inlé (Birmanie), ou la construction d'un paysage agricole et touristique original », Projets de paysage [En ligne], 14 | 2016, mis en ligne le 21 septembre 2016, consulté le 31 juillet 2020. URL : http://journals.openedition.org/paysage/8291 ; DOI : https://doi.org/ 10.4000/paysage.8291

Ce document a été généré automatiquement le 31 juillet 2020.

Projets de paysage 


\title{
Les jardins flottants du lac Inlé (Birmanie), ou la construction d'un paysage agricole et touristique original
}

\author{
The Floating Gardens of Lake Inle (Burma), Or the Building of an Original \\ Agricultural and Touristic Landscape
}

Martin Michalon

1 Le lac Inlé, au centre est de la Birmanie, est célèbre pour l'ethnie intha, qui vit sur ses eaux et qui a su mettre en place un mode de vie original, entre pêche, agriculture flottante et artisanat. Le dynamisme de cette population a présidé à la création d'un paysage original, mêlant villages sur pilotis, jardins flottants, pêcheurs, etc. Cependant, une étude plus approfondie de la région révèle que ces paysages, loin d'être intemporels, sont en mutation rapide, notamment du fait d'une intégration à la mondialisation plus précoce et plus intense que les images d'Épinal touristiques ne peuvent le laisser penser.

2 En raison de la junte militaire au pouvoir jusqu'en 2010, le pays ne s'est ouvert que récemment au tourisme, avec une première phase d'ouverture en 1996, et une seconde en 2011. Au fur et à mesure de l'entrée du pays dans le marché touristique, les acteurs du secteur se sont emparés de la région et ont transformé les paysages et leurs habitants en produits touristiques à travers un ensemble de discours souvent folkloristes, célébrant l'« authenticité » du pays. Si l'ensemble du paysage d'Inlé est mentionné dans cet article, nous nous focaliserons cependant sur les jardins flottants, traits visuels majeurs du territoire local.

3 Cet article présente le résultat de nos recherches de terrain, menées en février 2014 dans le cadre d'un master 2 de géographie (Michalon M., 2014) et de février 2015 à juin 2016 dans le cadre d'une thèse de doctorat actuellement en cours. Étant donné la méfiance de la junte militaire birmane par rapport à la recherche occident ale, le 
naufrage de la recherche birmane à cette époque et l'incurie de l'administration locale, le chercheur ne peut s'appuyer que sur de très rares sources bibliographiques, souvent biaisées, voire totalement fausses. Il n'est donc pas toujours possible de capitaliser sur les travaux antérieurs. Notre travail se fonde donc en partie sur une utilisation précautionneuse de sources coloniales, de travaux birmans des années 1990 et 2000, de rares travaux de chercheurs occidentaux ainsi que sur des rapports d'ONG. Cependant, sur un sujet aussi peu étudié, les sources secondaires sont rares, et l'essentiel de nos informations provient d'observations directes sur le terrain et d'entretiens semistructurés avec des dizaines d'acteurs aux profils très variés: exploitants de jardins flottants, vendeurs d'intrants, grossistes en tomates, pêcheurs, touristes, pilotes de bateaux, hôteliers, etc.

4 Alors que le lac Inlé est aujourd'hui intégré dans plusieurs projets de développement, les ONG s'appuient régulièrement sur des sources scientifiques erronées et sur des entretiens avec des responsables politiques ou de la société civile, qui ont leurs propres stratégies politiques ou économiques. En revanche, lorsque l'on s'intéresse aux interlocuteurs du quotidien, à la sphère du foyer, le résultat nous semble plus fiable, bien que le travail soit plus fastidieux, la montée en généralité plus difficile, les stratégies et discours partisans toujours présents. Dans une société de l'oralité, nos conversations se sont souvent heurtées à un manque d'informations, de dates, de statistiques précises. Il nous a donc fallu multiplier les entretiens pour faire émerger des phénomènes et les quantifier autant que possible.

5 Ce travail de terrain, très empirique et précautionneux, fait de recoupements systématiques et d'un recul critique permanent, nous a permis de répondre aux questions suivantes: dans quelle mesure les jardins flottants du lac Inlé sont-ils le support d'une monoculture intensive construite par des jeux d'acteurs complexes, et s'intégrant dans les échelles spatiales et les mécanismes de la mondialisation? Malgré cette connexion aux dynamiques de la mondialisation, comment les jardins ont-ils été érigés par les acteurs du tourisme comme symbole d'une «tradition » intemporelle et folklorique, atout de la Birmanie sur le marché du tourisme international ?

\section{Le lac Inlé : un lac de montagne fortement anthropisé}

6 Le lac Inlé est le second plus important de Birmanie, après le lac Indawgyi, situé dans l'état Kachin, au nord du pays. Il est situé à l'ouest du plateau Shan, qui couvre la majeure partie de l'Est du pays. Situé à une altitude de 890 mètres, il est encadré par des chaînes de collines nord-sud culminant à environ 2000 mètres. 
Figure 1. Le lac Inlé dans le contexte birman

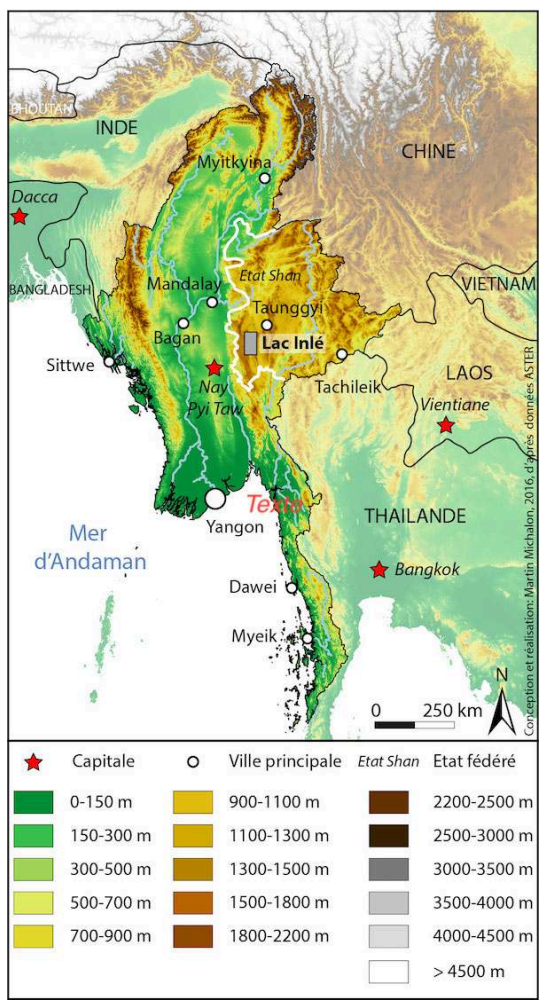

Figure 2. Inlé : un lac de montagne

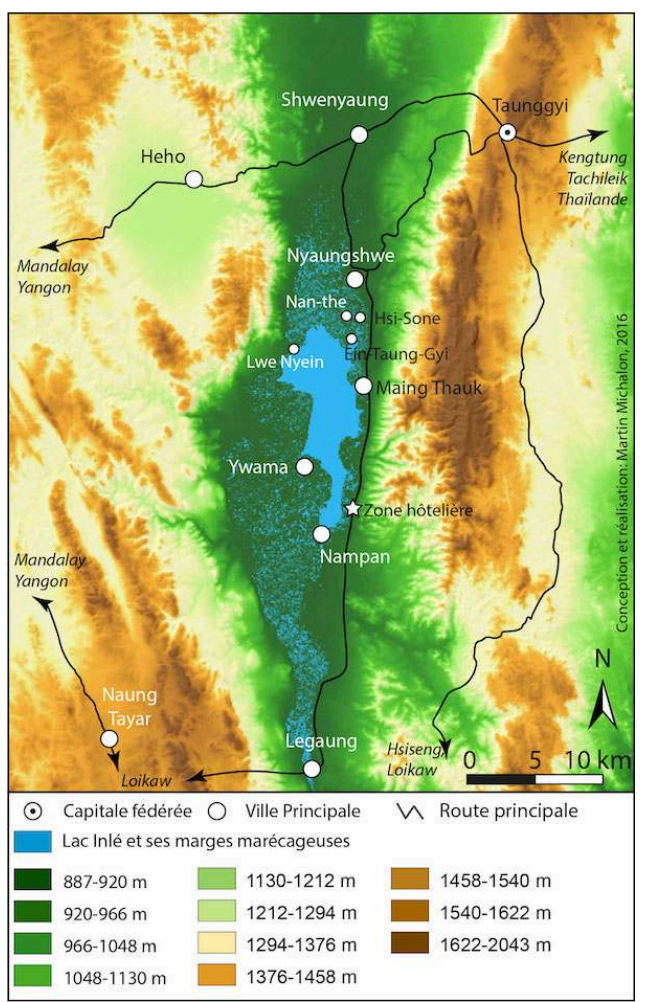

7 À l'instar de Takahisa Furuichi (2008), nous appelons « lac Inlé » toute l'étendue en eau depuis le village de Nyaung Shwé, au nord, à celui de Nampan, au sud. Ce dernier 
marque l'endroit où le lac Inlé se resserre pour devenir la rivière Nam Pilu, qui se jettera ultérieurement dans la Salouen. Nous prenons ici nos distances avec certains chercheurs et certains départements de l'administration birmane, qui considèrent que «le lac » s'étend jusqu'au village de Legaung, $20 \mathrm{~km}$ plus au sud.

Le bassin-versant du lac Inlé présente une superficie de $3800 \mathrm{~km}^{2}$, et comporte 30 rivières qui ont un débit cumulé de 1,35 milliard de mètres cubes/an (Furuichi, 2008). Le lac est soumis au régime de mousson, qui fait osciller son volume entre 70 et 238 millions de mètres cubes selon les années et les saisons, avec un volume moyen de 158 millions de mètres cubes (ibid.).

D'après des images satellites Google Earth de février 2014, les plus grandes dimensions de la zone en eau libre sont de $17 \mathrm{~km}$ de long et 5,1 km de large, pour une aire de $46 \mathrm{~km}^{2}$. Cependant, cet ensemble ne représente qu'une partie du lac, qui comporte également de vastes étendues de marécages et de jardins flottants. D'après nos mesures sur ces mêmes images, corrélées à des entretiens avec des paysans locaux ${ }^{1}$, la surface inondable totale serait d'environ $120 \mathrm{~km}^{2}$.

10 Si l'on se penche plus avant sur cette région, le lac Inlé apparaît comme très fortement anthropisé. En effet, sa surface est parsemée de plus de 200 villages et hameaux sur pilotis qui rassemblaient en 2005 pas moins de 25000 habitants, auxquels s'ajouteraient 75000 personnes dans les villages sur les marges du lac (NCEA, 2006), appartenant pour la plupart à l'ethnie intha. Cette population, qui trouverait ses origines dans la région de Dawei, au sud du pays, se serait implantée dans la région au xive siècle (Robinne, 2000), contrastant avec les Shans, Danu, Taungyo et Pa-O qui dominent dans les collines. Revendiquant un lien symbiotique avec le lac Inlé (en birman, « in » signifie «lac», et «tha» signifie le/les fils), les Intha ont su mettre en place un mode de vie très particulier, qui a profondément transformé le paysage de la région, jusqu'à en faire l'un des symboles de la Birmanie.

11 Habitant dans des maisons de teck ou de bambou, ils vivent d'une polyactivité étudiée en détail par Lucien Bernot (1972). D'une part, ils sont des artisans réputés : tissage, bijouterie, vannerie, fabrication de cigares, charpente... D'autre part, montés sur de petits canoës qu'ils propulsent en ramant avec la jambe, ils pratiquent la pêche, traditionnellement à l'aide d'un vaste filet (le saung) tendu sur une armature conique de bambou. Cependant, du fait de la baisse des prises de poisson, cette pratique tend aujourd'hui à s'effacer au profit des filets classiques en matériaux synthétiques (Okamoto, 2012; Michalon, 2014). Enfin, ils ont mis en place un étonnant système de culture maraîchère sur des îles flottantes formées à partir de jacinthes d'eau, qui produisent des tomates renommées dans tout le pays. Ces jardins flottants sont aujourd'hui une dimension incontournable du paysage d'Inlé, l'un des principaux moteurs de sa transformation, et un attrait touristique majeur.

\section{Les jardins flottants du lac Inlé, ou la création d'un paysage original}

12 Les jardins flottants, localement appelés ye-chan (Thi Dar Win, 1996), sont une forme d'agriculture tirant profit de la prolifération des jacinthes d'eau (Eichhornia crassipes) sur le lac. Ces dernières forment des masses flottantes, que les vents et les courants poussent vers les marges du lac. Là, les racines s'entremêlent et captent les sédiments 
en suspension dans l'eau. Il faut compter entre vingt et trente ans pour former un substrat flottant dense, épais d'environ un mètre, prêt à être découpé en bandes d'environ 1,5 mètre de large et de plusieurs dizaines de mètres de long. Ces dernières sont ensuite remorquées vers leur destination: le champ familial, délimité par une ceinture d'îles flottantes et de bambous. Là, des piquets de bambou les maintiennent à leur emplacement tout en leur permettant de coulisser verticalement pour suivre les fluctuations du niveau de l'eau (Bruneau et Bernot, 1972). La verticalité des milliers de piquets jalonnant les eaux est une dimension importante du paysage de jardin flottant. Les ye-chan sont alors désherbés, puis recouverts d'une couche de limon extrait du fond du lac, d'une couche d'algues récoltées sur ses eaux, et d'une ultime couche de limon, sur laquelle sont repiqués les jeunes plants de tomates en provenance de pépinières qui jouxtent la maison familiale (Bruneau et Bernot, 1972; Michalon, 2014). Cette technique, intensive en main-d'œuvre et permettant d'obtenir des rendements élevés, s'est répandue sur le lac, jusqu'à devenir un élément structurant de ses paysages et de son économie.

Figure 3. Les étapes de la fabrication des jardins flottants
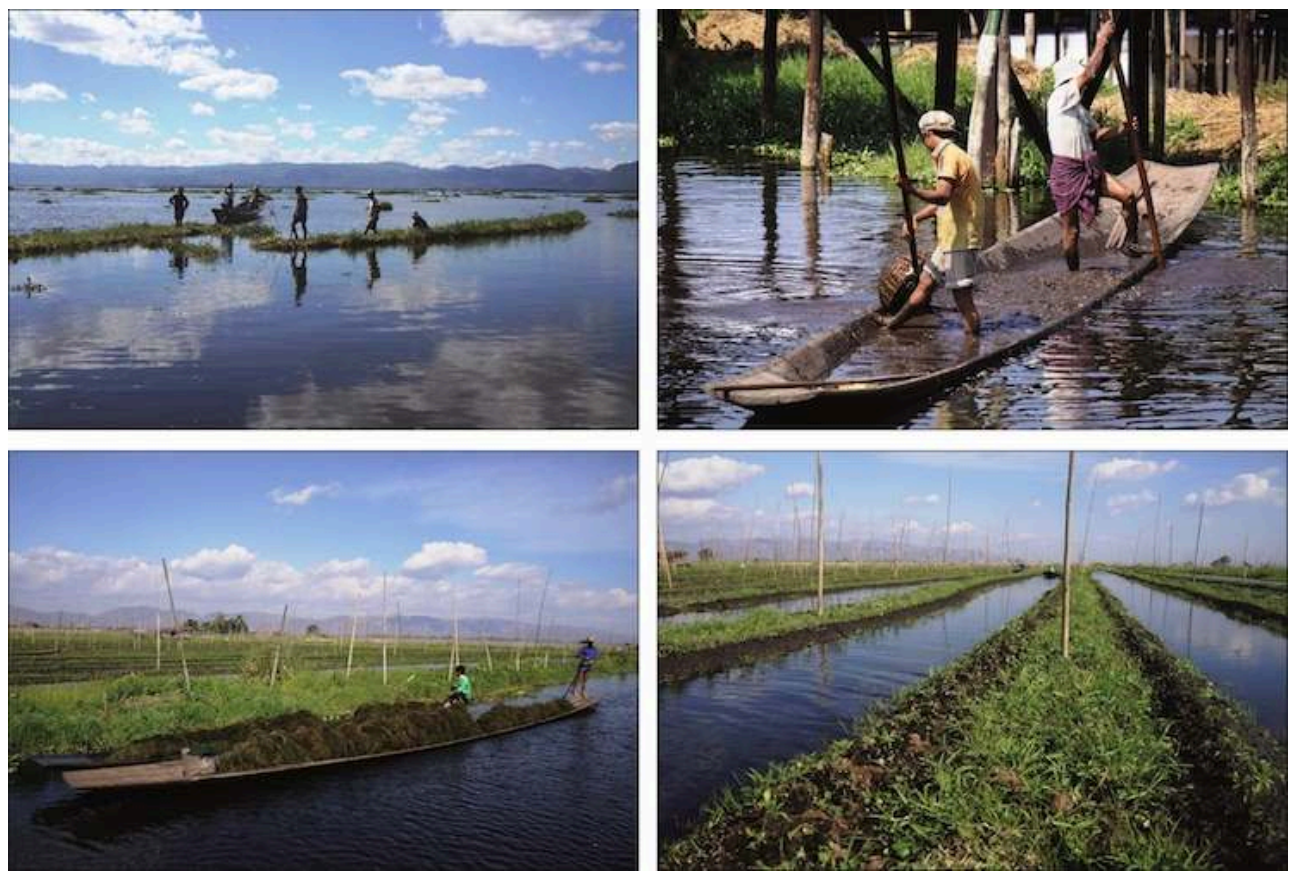

De haut en bas et de gauche à droite : le découpage des îles flottantes sur le lac ; la collecte de limon ; la récolte d'algues à la surface des eaux; la plantation des jeunes plants de tomates.

Source: Martin Michalon, de 2014 à 2016.

13 Cependant, contrairement à ce qui est présenté aux touristes, l'agriculture flottante dans sa forme actuelle n'est qu'un phénomène assez récent, ne serait-ce que parce qu'il est établi que la jacinthe d'eau, si importante dans la composition des ye-chan, n'a fait son apparition en Birmanie que dans les premières années du $\mathrm{xx}^{\mathrm{e}}$ siècle (Bernot et Bruneau, 1972). Certes, lors de son voyage dans la région en 1896, Robert Gosset Woodthorpe décrit de manière très anecdotique, le temps d'une demi-phrase, des «jardins flottants ${ }^{2}$ sur lesquels on cultive des tomates, des pastèques, des calebasses et des feuilles de bétel ». 
Figure 4. Extrait de "Some account of the Shans and hill tribes of the States on the Mekong "

The Inle Lake is a vast expanse of water, 13 miles long and about 4 miles wide at its upper end, but narrowing towards the south. It is nowhere very deep, and the bottom is overgrown with long and tangled weeds, which rise nearly to the surface. The lake dwellings of the Inthas, an amphibious tribe said to have been originally brought as slaves from the province of Tavoy, rise on piles out of the water in groups near the edge, and floating gardens, on which are grown tomatoes, water melons, gourds and the pan leaf vine, dot the surface of the lake around them. Many large villages are seen on either shore; monasteries and pagodas, built on the extremities of the spurs, running into the lake, are reflected in its placid depths.

Source: R. G. Woodthorpe, 1897, voir lignes 8 et 9 .

Cependant, dans les Gazetteer of Upper Burma and the Shan States de 1901, l'un des ouvrages les plus détaillés sur la Birmanie coloniale, nulle mention n'est faite des yechan dans les treize pages dédiées à la région d'Inlé, et la tomate n'apparaît pas dans la liste des 58 biens courants observés au marché de Nyaung Shwé, ce qui laisse à penser au caractère marginal de cette pratique (Scott et Hardiman, 1901).

Ce n'est qu'en 1918 que Nelson Annandale décrit assez précisément une forme d'agriculture flottante sur le lac, mais d'envergure très réduite (Annandale, 1918) : certaines familles du lac cultivent bien quelques rangs de légumes autour de leur maison, mais pour un usage purement familial.

Figure 5. " Jardins flottants dans la zone marginale du lac, montrant des cultures d'oignons nouveaux, de tomate et de concombres, ces derniers poussant sur des treilles. ”

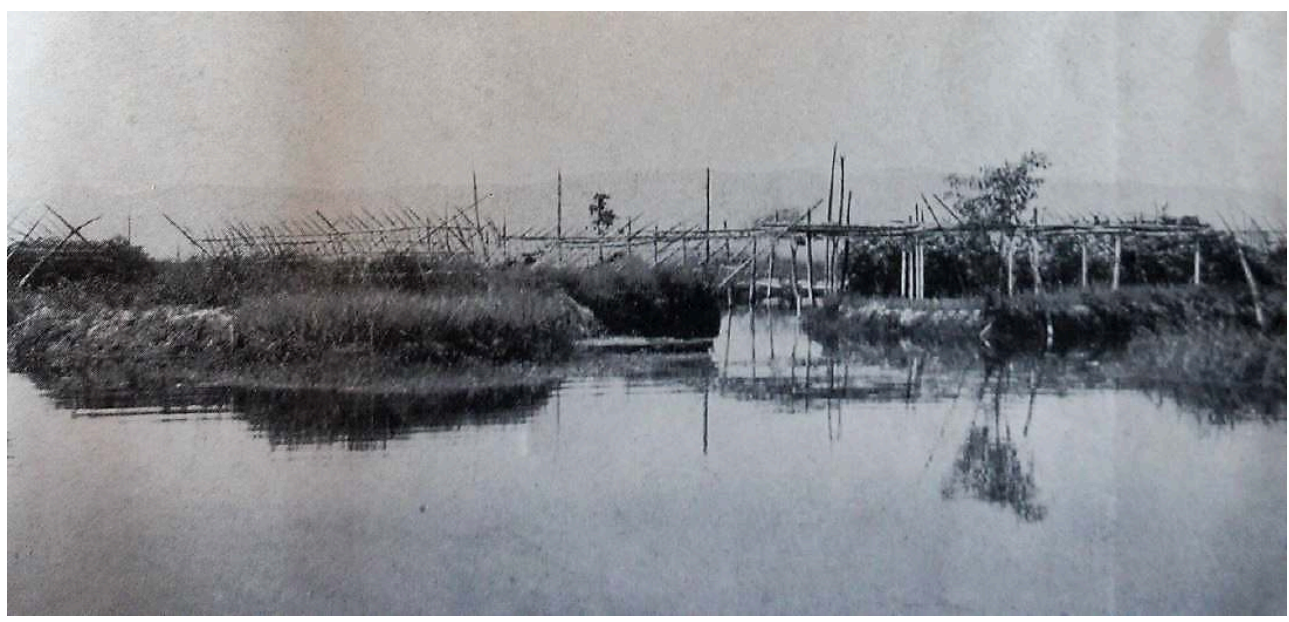

Source : Annandale, 1918.

La Seconde Guerre mondiale paraît marquer une étape clé dans la diffusion des cultures flottantes. En 1968, un anthropologue américain précise que «la méthode [d'agriculture flottante] est connue depuis au moins 50 ans, et peut-être bien plus, mais la vingtaine de villages qui aujourd'hui se sont spécialisés dans la tomate n'ont commencé à le faire qu'à partir des années 1930, et surtout après la Seconde Guerre mondiale » (Sturtevant, 1968). Cette thèse semble trouver sa confirmation dans les 
rares témoignages que nous avons pu recueillir auprès de personnes âgées de la région ${ }^{3}$ : durant la guerre, une partie de la population des terres avait en effet trouvé refuge sur le lac (Daw Than Htay, communication orale). On a donc tout lieu de penser que les jardins flottants ont été agrandis, et leur usage systématisé pour répondre à cet afflux de population.

17 L'expansion de l'agriculture flottante connaît un nouveau sursaut dans les années 1960, pendant lesquelles la junte militaire améliore le réseau de communication dans le pays, créant un débouché pour les produits du lac (Robinne, 2000), et notamment la tomate, qui est depuis des décennies le produit phare, avec $90 \%$ des surfaces cultivées aujourd'hui (Thi Dar Win, 1996). La tomate d'Inlé connaît un fort succès car le mode de culture propre au lac permet de s'affranchir des contraintes d'irrigation, et donc de la saisonnalité, et de tirer profit des cours élevés en saison sèche. En 1972, Lucien Bernot et Michel Bruneau décrivent ainsi une filière organisée, une activité qui marque les paysages et l'économie locale selon des modalités relativement proches de celles d'aujourd'hui.

Figure 6. « îles flottantes mises en place près des rives du lac »

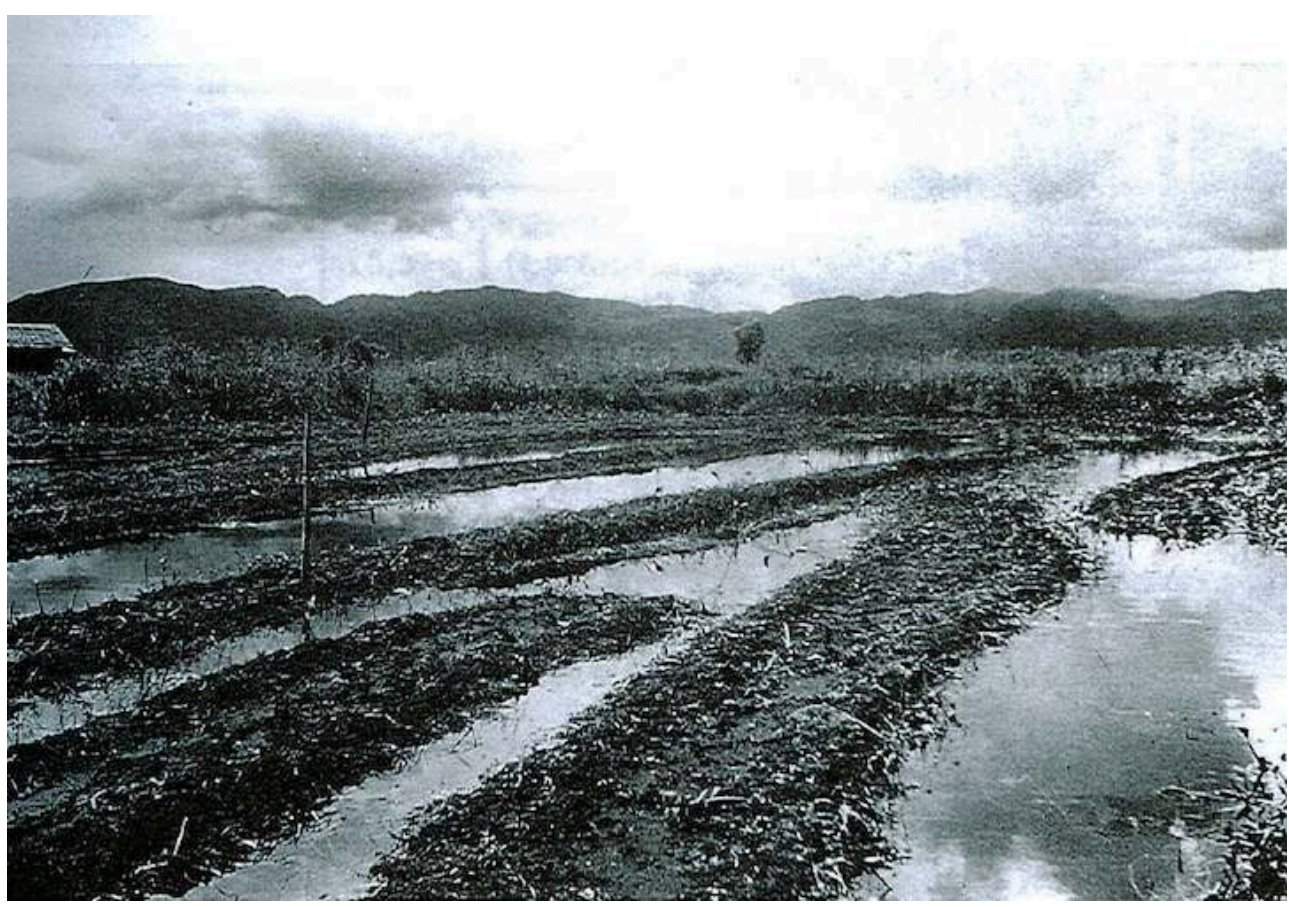

Source : Bernot et Bruneau M, 1972.

18 Une seconde impulsion est donnée lorsqu'en 1995, la junte militaire renonce à sa quête de "voie birmane vers le socialisme " et dissout les coopératives textiles et agricoles alors en vigueur. La première mesure prive de nombreuses familles des revenus textiles; la seconde libère l'initiative privée dans l'agriculture (U Sai Win, communication orale): les conditions sont donc réunies pour un nouvel essor des jardins flottants. D'après nos mesures effectuées sur des images Google Earth de 2014, ils couvriraient actuellement près de 3200 hectares, et occupent désormais une place prépondérante dans le paysage lacustre, sous la forme de vastes parcs maraîchers sur les marges du lac. 
Figure 7. Un « parc » de jardins flottants sur la rive ouest du lac, près du village de Kyun Gyi

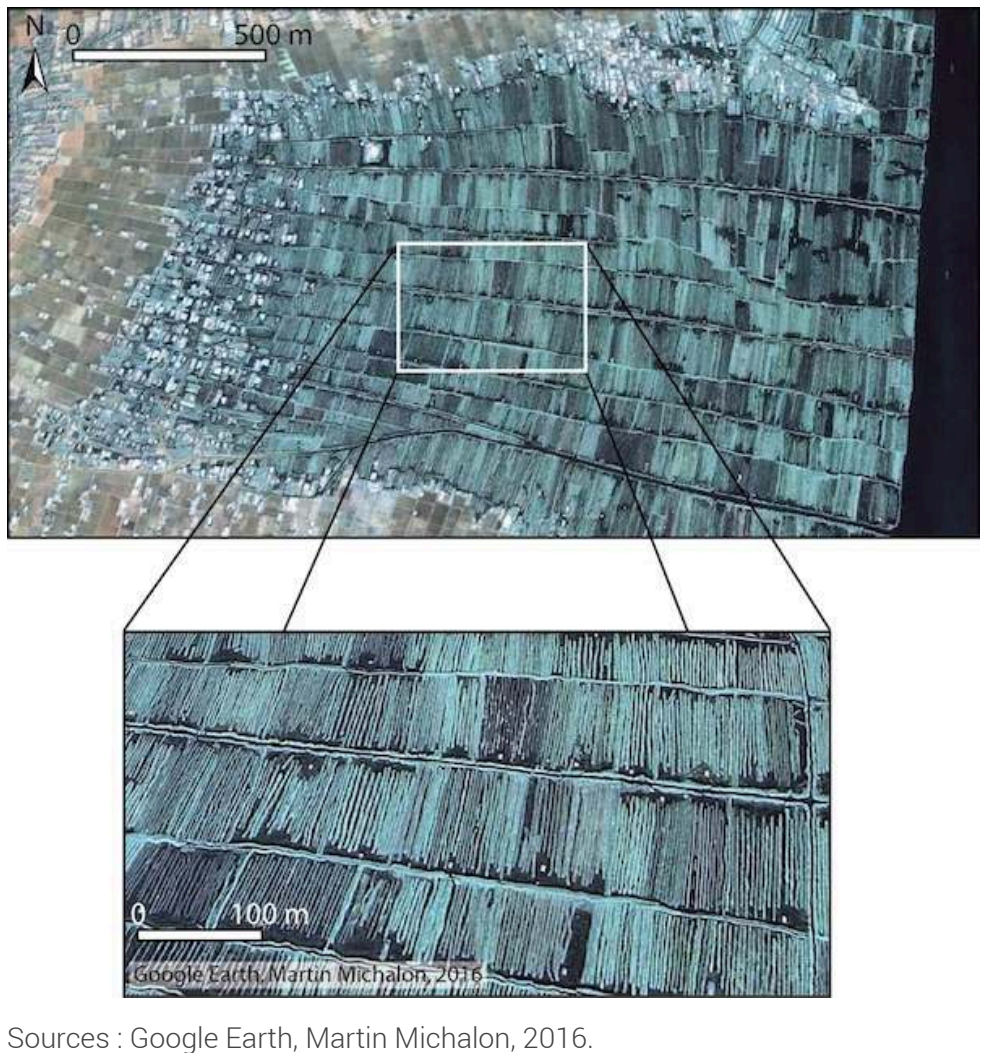

19 Contrairement à ce qui est souvent sous-entendu dans les guides touristiques ou dans les discours des acteurs touristiques, l'agriculture flottante (sous sa forme actuelle du moins, massive et commerciale) ne se perd donc pas dans les limbes du passé : c'est un phénomène récent. Il est également faux de la présenter comme une pratique folklorique, très artisanale, voire primitive, et surtout de la cantonner à une échelle uniquement locale : l'agriculture flottante d'aujourd'hui est en réalité très intensive, forte consommatrice d'intrants, et totalement intégrée dans les réseaux de la mondialisation économique.

\section{Loin de « la région hors du temps »: modernité d'un paysage agricole dynamique}

Il suffit de suivre un groupe de touristes sur le lac pour remarquer que le guide expliquera en détail la fabrication «traditionnelle » des îles flottantes (jacinthes d'eau, limon, algues)... mais passera sous silence l'utilisation de produits phytosanitaires, pourtant généralisée. Ainsi, tous les fermiers que nous avons rencontrés ont recours aux engrais de synthèse, et certains les utilisent depuis une vingtaine d'années, voire, dans un cas, depuis une soixantaine d'années. La visite des dix entrepôts spécialisés dans la ville de Nyaung Shwé prouve que la région n'est en rien coupée du monde. Ainsi, en 2014, l'engrais le plus populaire dans la région était le Comet, fabriqué en Allemagne. En 2016, certains magasins mettent plutôt en avant les produits de la firme suisse Eurochem, auxquels il faut ajouter ceux des compagnies asiatiques : les Chinois sont très présents (compagnies White Pearl ou Weng Feng), tout comme l'Indonésien 
Pupuk. Les fermiers font également un usage systématique des pesticides, avec des conséquences sanitaires et environnementales potentiellement inquiétantes (May Lwin Oo et al., 2010).

La filière des semences contraste elle aussi avec l'image d'une "région hors du temps » et d'une pratique culturale rudimentaire : depuis des décennies, des semences hybrides sont utilisées, avec des changements fréquents pour tirer profit des améliorations de rendement. Ainsi, il y a 13 ans, le fermier Ko Aung Win utilisait la Typhoon 387 (commercialisée par le Thaillandais Chia Tai), avant de se convertir à la Red Gem de Monsanto, et d'adopter, il y a 3 ans, la Inlay 019, création de la multination ale EastWest Seed. Lire l'étiquette de ces sachets de graines, c'est retracer une véritable cartographie de la mondialisation agricole, selon le principe de la division internationale du travail. La semence Sahara 711 de la marque Seminis est ainsi fabriquée en Inde par Monsanto Thaïlande, avant d'être importé à Yangon par le Birman Seeds Energy.

L'utilisation généralisée de semences hybrides, d'engrais de synthèse et de pesticides, ainsi que d'une importante main-d'œuvre agricole génère des rendements élevés: 90000 tonnes de tomates seraient ainsi récoltées annuellement sur le lac (IID, 2012). Si l'on considère que tous les fermiers font deux cycles de récoltes par an, ce qui est le cas de la plupart d'entre eux, ils récoltent donc 45000 tonnes/cycle sur 3180 hectares de jardins flottants, soit un rendement de 14 tonnes/hectare. Or, d'après nos mesures satellites, $40 \%$ d'un ye-chan est composé d'eau (canaux de circulation entre les îles flottantes). Par conséquent, si l'on considère le rendement par surface de terre émergée, les jardins d'Inlé produisent 23,3 tonnes/hectare, soit sensiblement plus que la moyenne du Sud-Est asiatique, qui est de 16,7 tonnes/hectare (http://www.fao.org/). La culture des ye-chan apparaît donc comme une pratique intensive, pleinement intégrée dans les réseaux de la mondialisation et de l'innovation agronomique, bien loin de l'image folklorique qui en est souvent donnée.

Si l'on se penche sur l'aval de la filière, c'est-à-dire sur les circuits de commercialisation des tomates, une même logique apparaît. Les tomates récoltées sur le lac sont stockées dans de vastes entrepôts de Nyaung Shwé, appartenant à de puissants grossistes. Après une période de stockage dans l'attente du meilleur cours, elles sont expédiées hors de la région. Au milieu des années 1990, la région envoyait environ 75 tonnes de légumes par jour en haute saison, principalement à destination de la Birmanie centrale, dans la région de Mandalay (Thi Dar Win, 1996). Depuis, les volumes ont considérablement augmenté (jusqu'à 480 tonnes par jour), et l'éventail des destinations s'est considérablement élargi : les camions desservent toujours la Birmanie centrale, mais désormais également la région de Yangon, au sud du pays (Michalon, 2014).

Cet élargissement spatial des réseaux de distribution s'est basé sur une double mobilité d'acteurs commerciaux. D'une part, les grossistes de la région d'Inlé s'appuient sur des réseaux d'interconnaissances à base familiale ou ethnique dans les régions de destination: par exemple, $U$ Aung Aung ne fait affaire qu'avec son frère, installé à Mandalay, et son neveu à Yangon. D'autre part, des acheteurs du Sud du pays (région de Yangon) et de Birmanie centrale (région de Mandalay) s'installent désormais à Nyaung Shwé, du moins de juin à octobre, qui constitue la principale période de vente.

Cependant, si l'amarrage à la mondialisation a profité dans un premier temps à l'agriculture flottante, elle la fragilise aujourd'hui: l'amélioration des télécommunications et des infrastructures de transport, l'apparition de nouvelles zones 
de production dans le Sud du pays et les importations de tomates thaillandaises bon marché génèrent une baisse des prix et une instabilité chronique des cours (U Aung Aung, communication orale). De nombreux exploitants de ye-chan doivent désormais faire face à une concurrence accrue, et sont donc engagés dans une course au rendement (Michalon, 2014), d'autant que la sédimentation accélérée du lac menace les ye-chan (Michalon, à paraître).

D'un point de vue économique et démographique, la région d'Inlé s'est donc largement transformée, et fait aujourd'hui figure de moteur de l'état Shan : alors que le township de Nyaung Shwé comptait environ 86000 habitants en 1969 (Bernot et Bruneau, 1972), il en comptait 189000 en 2014 (Ministry of Immigration and Population, 2015), soit un taux de croissance proche de $1,8 \%$ par an. Il est aujourd'hui le troisième plus peuplé des 55 townships de l'état Shan, après ceux de Taunggyi et de Lashio (ibid.). Par conséquent, il apparaît de manière assez claire que la région d'Inlé et son paysage si typique ne constituent en rien un isolat géographique et temporel. C'est au contraire une région pleinement intégrée à toutes les échelles, un territoire dynamique et réactif que les jeux d'acteurs ont su insérer dans les grandes dynamiques. La plus récente, et aujourd'hui la plus massive : le tourisme.

\section{La construction d'un idéal touristique}

Le tourisme est un phénomène récent en Birmanie. En effet, sous la coupe d'une junte militaire "teintée de xénophobie paranoïaque ( Egreteau, 2009) de 1962 à 2011, la Birmanie est longtemps restée en marge de l'explosion touristique de l'Asie du Sud-Est. En 1996, le gouvernement lance une Visit Myanmar Year qui, malgré des résultats mitigés (250 000 visiteurs, dans Henderson, 2003), marque les premiers pas du pays dans le tourisme moderne. Les années suivantes sont cependant heurtées, entre appels au boycott touristique de la part de nombreuses ONG occidentales et d'Aung San Suu Kyi, icône de l'opposition alors assignée à résidence, répression sanglante de manifestations populaires (révolution safran de 2007) et catastrophe naturelle (cyclone Nargis de 2008).

En 2010, à la surprise générale, la junte militaire s'autodissout au profit d'un régime officiellement civil, qui accède au pouvoir en 2011. Les flux touristiques connaissent alors une augmentation spectaculaire, passant de 791000 visiteurs en 2010 à 4,68 millions en 2015, soit un taux de croissance de $43 \% / \mathrm{an}^{4}$ (Ministry of Hotels and Tourism, 2011 et 2016). Selon toute probabilité, le phénomène devrait connaître une nouvelle accélération cette année, avec l'accession triomphale au pouvoir de la Ligue nationale pour la démocratie, parti d'Aung San Suu Kyi, en avril 2016. Dans ce contexte, et face à la concurrence des autres destinations asiatiques, les acteurs touristiques jouent de cette longue fermeture, et mettent en avant la "tradition ", l'intemporalité de la Birmanie, cette « bulle temporelle » qui se révèle au monde.

Si le lac Inlé est absent des guides de voyage de l'époque coloniale (Murray's Guidebook 1901, 1949, 1962), il émerge comme l'une des rares destinations touristiques à l'époque de la junte. Dans l'ouvrage Birmanie, de Guillon et Delachet (1975), les auteurs donnent une description assez approfondie de l'État Shan, et la seule mention de l'industrie touristique de l'État concerne Inlé : « Les touristes peuvent rendre visite aux Intha sur leurs îles flottantes. »Cependant, jusqu'au milieu des années 1990, le tourisme reste anecdotique. Un premier sursaut se produit lors de la Visit Myanmar Year de 1996. Le lac 
Inlé figure parmi l'éventail encore très étroit de destinations autorisé par la junte, proposé lors des foires internationales (Kyi Kyi Hla, 1996) et rappelé dans les publications officielles (Thaw Kaung, 1996).

Cependant, le réel essor du tourisme à Inlé a lieu dans le sillage de la transition démocratique de 2011. Ainsi, la quasi-totalité des acteurs du tourisme à Inlé (hôteliers, restaurateurs, propriétaires d'agences de voyages, pilotes de bateaux, chauffeurs de taxi, etc.) s'accordent à dire que l'année 2012 fut une année charnière. Dans la foulée, l'année 2013 fut exceptionnelle, et nombre de touristes durent loger dans les monastères des environs, faute de chambres d'hôtel disponibles.

Les visiteurs découvrent alors cette région à travers deux symboles: d'une part, les jardins flottants, composante majeure du paysage si caractéristique de la région; d'autre part, les pêcheurs intha, leur façon si particulière de ramer et de pêcher, qui en font les gardiens de l'identité des lieux. Ces derniers occupent une place absolument déterminante dans l'imaginaire touristique birman: si les temples de Bagan sont la vitrine architecturale, patrimoniale de la Birmanie, le pêcheur intha en est le visage humain. Il apparaît ainsi en couverture du guide Lonely Planet 2011 et du Petit Futé 2014, sur les sites Internet d'absolument tous les tour-opérateurs intervenant dans le pays, sur celui de la Myanmar Tourism Federation, chargée de promouvoir la destination Birmanie à l'étranger ; et quand l'on saisit «Inle lake » sur Google Images, 146 images de pêcheurs apparaissent en première page (400 résultats), soit $36,5 \%$.

Cette visibilité est largement le résultat de jeux d'acteurs locaux comme extérieurs, que nous avons pu étudier au cours d'entretiens approfondis sur le terrain: depuis une dizaine d'années, une vingtaine de pêcheurs a été encouragée par les guides touristiques à poser devant les appareils photo des visiteurs, moyennant un pourboire.

Figure 8. Mise en scène de la « tradition » pour le tourisme : les tourist fishermen du lac Inlé

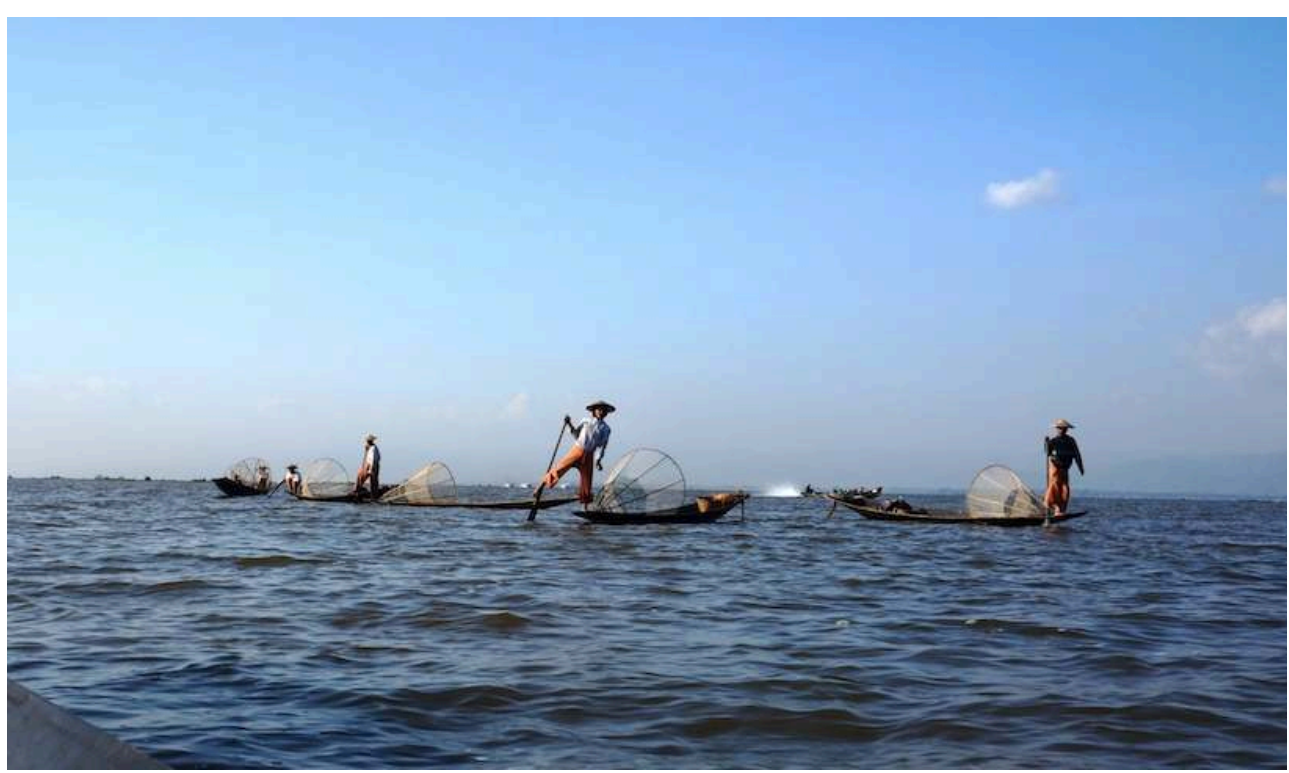

Source : Martin Michalon.

Arborant des tenues «traditionnelles » suggérées par des guides qui veulent répondre aux attentes de leurs clients, ils manipulent de manière spectaculaire et largement 
inventée le saung, filet conique pourtant devenu quasiment inutile auprès des «vrais pêcheurs ».

Figure 9. La manipulation spectaculaire du saung

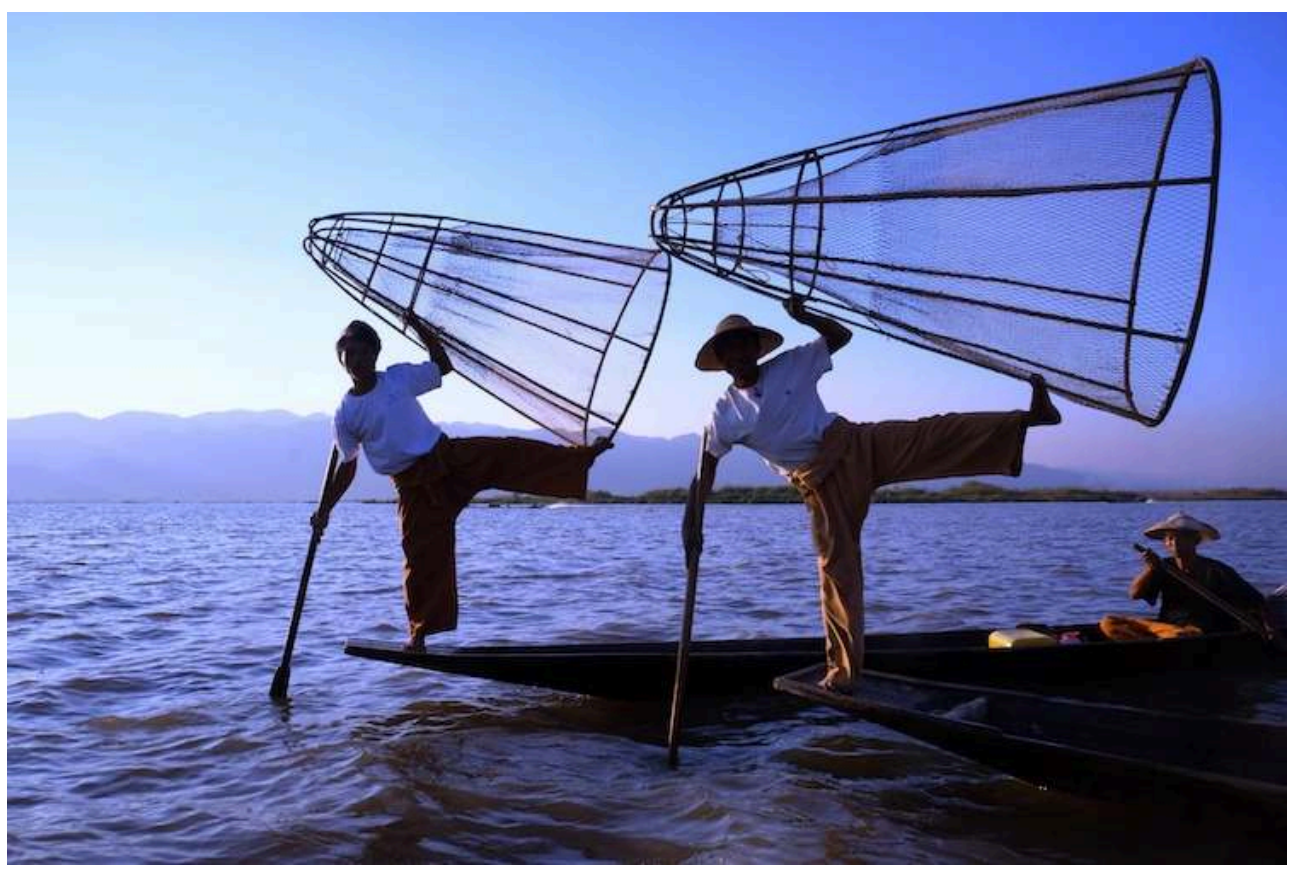

II s'agit d'un geste tout à fait inventé qui ne représente en aucun cas les techniques de pêche réelles. Source : Martin Michalon

Il ne faudrait cependant pas céder à l'image de pêcheurs passifs, manipulés par des agents extérieurs. La construction du personnage du «pêcheur intha » est en effet un processus dialogique, dans lequel les populations locales ont leurs propres stratégies et intérêts, et savent jouer des codes touristiques et des attentes des visiteurs, attitude bien résumée par Vincent Coëffé et al. (2007) : "Les acteurs de la sphère touristique sont capables d'actualiser, reformuler, combiner les éléments en leur donnant une nouvelle forme qui permet bien souvent de maintenir des éléments de culture moribonds. » Cette mise en scène de soi par cette poignée de " pêcheurs intha » a donc permis la construction d'un symbole régional, aisément reconnaissable.

Si les pêcheurs ont su folkloriser des traits culturels, des costumes, des gestes « en cours de disparition » (quand ils ne sont pas inventés), les fermiers n'ont pour l'instant pas fait cette démarche, en raison des trajectoires économiques divergentes de ces deux secteurs. Ainsi, la construction de la figure du "pêcheur intha" trouve son origine dans la crise du secteur de la pêche à la fin des années 2000 (Okamoto, 2012) : d'après nos entretiens, le tourisme est apparu comme une porte de sortie bienvenue pour des pêcheurs qui ont donc joué le jeu du tourisme et ont su exploiter les attentes d'acteurs extérieurs, guides comme touristes. De manière plus générale, il est intéressant de constater que c'est lorsqu'une activité s'efface comme pratique quotidienne, banale, qu'elle est érigée en patrimoine, en "tradition». De son côté, malgré ses récentes difficultés, l'agriculture flottante est encore un secteur dynamique grâce à son intégration à des réseaux nationaux et régionaux; c'est pourquoi ses paysages et les pratiques associées ne font pas l'objet d'une folklorisation « de l'intérieur », venant des fermiers. 

dans les livres de voyage et les guides touristiques, compagnons incontournables des voyageurs. Plusieurs thèmes ressortent en effet de manière récurrente, à commencer par les temporalités longues, le caractère immémorial de l'agriculture flottante, les références à un passé lointain et volontiers mythique; second topos: l'admiration devant l'ingéniosité d'un peuple laborieux qui a su mettre sur pied un système cultural décrit comme unique.

L'ouvrage Bonjour la Birmanie (Holzbachova et Benet, 1996) convoque ainsi ces deux thèmes simultanément :

«On raconte qu'au XII siècle, le roi Alaungsithu qui voulait contrôler la région a forcé les habitants de Dawei (Tavoy) à venir s'installer sur le lac, bientôt réduits à une forme d'esclavage et encerclés par les montagnards ennemis. [...] Puis ils décidèrent de se libérer des montagnes ennemies en allant conquérir leur liberté sur l'eau et construire des villages lacustres. Il fallait manger. [...] Ils cultivent toute l'année [...] des îles flottantes. Ce sont des couches végétales d'un mètre d'épaisseur constituées depuis des siècles à base de plantes qui flottent à la surface de l'eau [...] [qu']on recouvre ensuite de boue et d'argile. Et on plante. Et ça pousse. »

En 1998, le gouvernement birman, cherchant à soutenir la croissance du tourisme, publie dans son magazine officiel Myanmar Perspectives un article sur les «Industrieux Intha du lac Inlé5 » (Thaw Kaung, 1998), affirmant que :

«... les touristes des quatre coins du monde viennent au lac Inlé pour observer ces Intha si uniques, population lacustre qui s'est si bien adaptée à son extraordinaire mode de vie qu'elle vit en totale harmonie avec son environnement, grâce à une économie aquatique et agricole durable. [...] Ils ont une méthode tout à fait unique de culture des fleurs et des légumes sur de petites îles flottantes artificielles qui ne peut être observée que sur le lac Inlé, et nulle part ailleurs dans le monde.»

Preuve que les jardins flottants continuent à fasciner au fil des ans, Le Guide du Routard 2006 fait l'éloge de la créativité des Intha :

« N'ayant pas à l'état naturel de réponses à leurs problèmes, ils ont cherché des solutions qui n'étaient pas dictées par l'évidence, loin de là. C'est ainsi qu'ils ont eu l'idée (géniale, il faut l'avouer) d'installer des jardins flottants sur les eaux du lac, faute de pouvoir cultiver la terre des rives. Les jardins flottants sont l'une des grandes curiosités du lac.»

Plus récemment encore, Le Petit Futé 2014 se livre à une description hasardeuse de la technique des ye-chan dans un imaginaire mêlant glaciers, coraux et temporalités longues pour décrire :

«... l'essor des jardins flottants, l'une des attractions touristiques du lac. [...] Ces jardins ne flottent que durant la saison des pluies, quand les eaux du lac sont gonflées par la fonte des glaciers. [...] À l'origine, c'est l'accumulation, pendant des milliers d'années, d'herbes et de coraux, qui forma des masses dérivantes d'humus qui se sont amalgamées sur environ un mètre d'épaisseur pour former des terrains flottants. »

41 Les tour-opérateurs se sont également emparés des jardins flottants et ont intégré ce mode cultural dans le discours qui domine actuellement le marketing de la « destination Myanmar » : l'intemporalité, les traditions, les mythes. Ainsi, l'agence Travels Myanmar affirme sur son site Internet :

«Le lac Inlé se situe dans les fraîches et verdoyantes collines de l'état Shan, une région d'une beauté et d'une diversité culturelle stupéfiantes. Depuis des siècles, les Intha ont développé un mode de vie unique autour de ce superbe plan d'eau. Ils 
vivent dans des maisons sur pilotis sur le lac, et cultivent même leurs légumes sur des jardins flottants ${ }^{6}$. » désormais, l'iconographie touristique prend littéralement de la hauteur pour s'éloigner du tumulte touristique de la surface. D'une part, le succès croissant des drones de loisir auprès des visiteurs et des documentaristes popularise les images spectaculaires de ces îles flottantes à perte de vue, sans les files de bateaux que l'on observe dans les étendues d'eau libre. D'autre part, la région compte désormais deux compagnies de montgolfières, ces fameux ballons qui sont désormais inséparables de l'imagerie des temples de Bagan, dans l'Ouest du pays. La compagnie Oriental Ballooning, installée dans la région depuis 2014, a ainsi effectué 61 vols de novembre 2015 à mars 2016, transportant 273 passagers. 


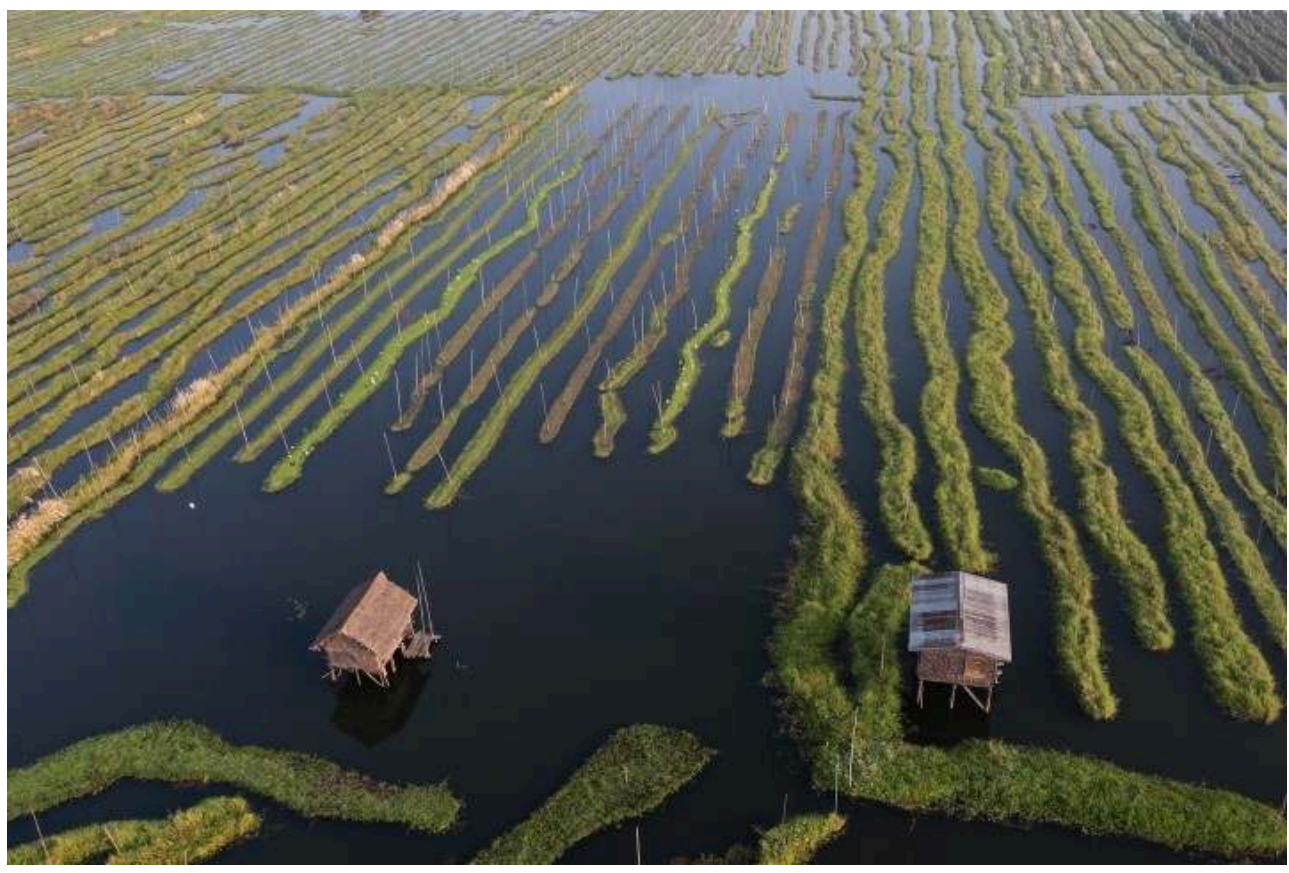

Source : d'après la compagnie Oriental Ballooning, https://www.orientalballooning.com/.

Bien que les drones et les montgolfières restent des pratiques négligeables d'un point de vue numérique, l'imagerie associée, grâce à ses qualités esthétiques et à son originalité, connaît une large diffusion sur Internet et dans les nombreux documentaires aujourd'hui tournés dans la région. Plusieurs vues aériennes figurent ainsi dans le documentaire Échappées belles: Birmanie, un nouveau départ, diffusé le 5 mars 2016 sur France 5, ainsi que dans la vidéo promotionnelle du tour-opérateur birman Myanmar Travel. Cette nouvelle imagerie touristique influe à son tour sur les représentations et les pratiques des visiteurs.

\section{Conclusion}

Les jardins flottants du lac Inlé font partie des incontournables touristiques de la Birmanie, pays qui fait valoir sur le marché mondial l'authenticité de sa culture et de ses paysages. Cependant, une étude plus approfondie sur le terrain révèle que, loin d'être un trait de terroir extrêmement localisé, cantonné à une échelle microlocale et figé dans la tradition, les jardins flottants incarnent finalement plus la modernité et les dynamiques de la Birmanie contemporaine que sa «tradition». Une analyse des systèmes d'acteurs met en évidence la construction exogène de cette représentation du jardin flottant : guides touristiques, tour-opérateurs, sites Internet, etc. À l'heure où la Birmanie s'insère dans la mondialisation, dans les flux touristiques, et connaît de profondes mutations, le discours de « l'authenticité menacée » se généralise. Il est donc très probable que toute activité, tout paysage considéré comme " prémondialisation ", "prétourisme", se verront parés des atours de la "tradition". C'est déjà le cas aujourd'hui dans une certaine mesure pour les ye-chan d'Inlé, mais nul doute que l'écart va s'élargir à l'avenir entre la réalité et les représentations de ce paysage original. 
Ce fossé risque d'être d'autant plus large que les difficultés actuelles de la filière maraîchère et la sédimentation croissante du lac posent la question de la pérennité de ces paysages à moyen terme. Si ces derniers ont toujours été dynamiques, évolutifs, et s'il n'est pas irrationnel d'évoquer leur disparition, cette perspective est en revanche difficilement envisageable par les acteurs du tourisme.

Dans quelle mesure, après avoir contribué à l'essor du tourisme, les ye-chan seront-ils sauvegardés par et pour ce dernier ? Quels acteurs vont intervenir dans ce mouvement de patrimonialisation, et quels discours vont-ils mettre en place, que va-t-on donner à voir des ye-chan, quelles représentations et pratiques seront sélectionnées?

Je tiens ici à remercier vivement mes directrices de thèse, Bénédicte Brac de la Perrière (CNRS, CASE) et Isabelle Sacareau (Bordeaux 3), ainsi que Yanni Gunnell (Lyon 2), qui fut mon directeur de mémoire de master 2 et qui me suit toujours de près. Je tiens également à témoigner de ma gratitude au pôle heSam pour son soutien financier des plus appréciables, ainsi que la région Rhône-Alpes, qui m'a soutenu durant mon travail de master 2. Une pensée également pour mes interprètes sur le terrain, sans lesquelles je n'aurais pas pu recueillir la parole des habitants de la région: Ma Thandar, Ma Khin Lay Way, Ma Cho Mee. Et une pensée toute spéciale pour Ma Catherine Hone Paing pour son précieux soutien.

\section{BIBLIOGRAPHIE}

Annandale, N., « Introductory account of the Inle Lake », Records of the Indian Museum, vol. XIV, Calcutta, 1918, p. 1-7.

Bernot L. et D., « Contribution à la linguistique et à l'ethnographie des Intha (Birmanie) », Asie du Sud-Est et monde insulindien. Bulletin du Centre de Documentation et de Recherche (CeDRASEMI), vol. 3/3, CNRS/EPHE, Paris, 1972, p. 1-8.

Bruneau, M. et Bernot, L., « Une population lacustre : les Intha du Lac Inlé », Journal d'agriculture tropicale et de botanique appliquée, T. XIX, n 10-11, octobre-novembre 1972, p. 402-441.

Coëffé V., Pébarthe H., Violier P., « Mondialisations et mondes touristiques », L’Information Géographique, vol. 71, 2007/2, p. 83-96.

Egreteau, R., « Pékin et l'épine birmane : La relation entre la Chine et la Birmanie à l'épreuve », Outre-Terre, $n^{\circ} 21,2009 / 1$, p. 259-265.

Furuichi, T., « Catchment Processes and Sedimentation in Lake Inle, Southern Shan State, Myanmar », Centre for Resource and Environmental Studies, the Australian National University, Canberra, 2008.

Guillon, E. et Delachet, C., Birmanie, Paris, Petite Planète, 1975, 189 p.

Henderson, J.-C., « The Politics of Tourism in Myanmar », Current Issues in Tourism, vol. 6, $\mathrm{n}^{\circ} 2$, 2003, p. 97-118.

Holzbachova, R. et Benet, P., Bonjour la Birmanie, Paris, Les Créations du Pélican, 1996, 141 p. 
Institute for International Development (IID), « Inlay lake conservation project: a plan for the future, In support of the Action Plan for Environmental Conservation and Sustainable Management of Inlay Lake 2010-2025 », Yangon, 2012, 121 p.

Kyi Kyi Hla, « Myanmar wins the Gulliver Révélation de l'Année Tourism Award », Myanmar Perspectives, vol 6/1996, Yangon, State Peace and Development Council (SPDC), p. 6-7.

Le Guide du routard, Myanmar/Birmanie, coordonné par Page, O., Paris, 2006-2007, 299 p.

Le Petit Futé, Myanmar/Birmanie, Paris, 2014, 406 p.

May Lwin Oo, Sutheerawatthana, P., Minato, T., « Comparison of Information Dissemination Methods in Inle Lake: A Lesson for Reconsidering Framework for Environmental Education Strategies ", Applied Environmental Education \& Communication, 9/1, 2010, p. 58-74.

Michalon, M., « Le jardinier et le pêcheur dans la mondialisation ; Le lac Inlé (Birmanie), une région en transition », mémoire de master 2 de géographie, université Lumière Lyon 2, 2014, $146 \mathrm{p}$.

Ministry of Hotel and Tourism, « Myanmar Tourism Statistics 2015 », Nay Pyi Daw, Ministry of Hotel and Tourism, 2016, 2 p.

Ministry of Hotel and Tourism, « Myanmar Tourism Statistics 2013 », Nay Pyi Daw, Ministry of Hotel and Tourism, 2014, 2 p.

Ministry of Hotel and Tourism, « Myanmar Tourism Statistics 2010 », Nay Pyi Daw, Ministry of Hotel and Tourism, 2011, 2 p.

Ministry of Immigration and Population, « Population by household type, sex and sex ratio, urban and rural », résultats du recensement de 2014, fichier excel téléchargé depuis www.themimu.info en avril 2015.

Murray's Guidebook (écrit par Rushbrook W.L.F), Murray's Travelbook to India, Burma and Ceylon, Murray's, Londres, 1962, $634 \mathrm{p}$.

Murrays' Guidebook (écrit par Hearn G.R.), Murray's Travelbook to India, Burma and Ceylon, Murray's, Londres, 1949, 792 p.

Murray's Guidebook (auteur inconnu), Murray's Travelbook to India, Burma and Ceylon, Murray's, Londres, 1901, $484 \mathrm{p}$.

National Commission for Environmental Affairs (NCEA), « Environmental Performance Assessment of Inlay Lake », National Performance Assessment and Subregional Strategic Environment Framework in the Greater Mekong Subregion, 2006, 333 p.

Okamoto I., " Coping and adaptation against decreasing fish resource: case study of fishermen in Lake Inle, Myanmar ", Institute of Developing Economies (IDE) discussion paper $n^{\circ} 329$, Chiba, 2012, 28 p.

Robinne, F., Fils et Maîtres du lac. Relations interethniques dans l'État Shan de Birmanie, Paris, CNRS éditions, 2000, $364 \mathrm{p}$.

Scott, J-G. et Hardiman, J.-P., « Gazetteer of Upper Burma and Shan States », partie II vol. III, Government Printing, Yangon, 1901, 456 p.

Sturtevant, W. C., "Agriculture on artificial islands in Burma and elsewhere », Proceedings of the VIIIth International Congress of Anthropological and ethnological Sciences, Tokyo et Kyoto, vol. 3, section B-10 « Material Culture », 1968, p. 11 à 13. 
Thaw Kaung, « The industrious Intha of Inle Lake », Myanmar Perspectives, vol. III-2/1998, Yangon, State Peace and Development Council (SPDC), p. 28-39.

Thaw Kaung, «Picturesque and enchanting Myanmar », Myanmar Perspectives, vol. V-12/1996, Yangon, State Peace and Development Council (SPDC), p. 62-65.

Thi Dar Win, «Floating island agriculture (ye-chan) of Inle Lake », mémoire de master, département de géographie, université de Yangon, 1996, 180 p.

Woodthorpe, R.G., « Some Account of the Shans and Hill Tribes of the States on the Mekong », The Journal of the Anthropological Institute of Great Britain and Ireland, vol. 26, 1897, p. 13-28.

\section{Sites Internet}

www.fao.org

www.orientalballooning.com

www.sandatour.com

www.travels-myanmar.com

www.unwto.org

\section{NOTES}

1. Ces recoupements sont nécessaires car des zones de rizières irriguées occupent les marges du lac, dans le prolongement de la zone d'inondation: lorsqu'elles sont en eau, elles accroissent visuellement (sur les images satellites) l'étendue du lac, alors que ce sont des zones distinctes.

2. En l'absence de jacinthes d'eau, ces jardins devaient être supportés par d'autres espèces de plantes aquatiques ou des radeaux de bambou.

3. Dans une société où l'écrit n'a longtemps eu qu'une place marginale, nous avons pu remarquer que les souvenirs remontent très rarement au-delà de deux générations. Par conséquent, des faits vieux de 60 ans sont considérés comme très anciens et se perdent dans les brumes du passé. Ces temporalités spécifiques sont donc à tenir en compte quand on se penche sur l'histoire locale. 4. Comme toutes les statistiques touristiques, ces chiffres recensent les étrangers traversant les frontières du pays pour plus de $24 \mathrm{~h}$ : ils prennent donc en compte tous les types de visas. Si l'on s'en tient au nombre de visas touristiques, la croissance est moins marquée : 201000 personnes en 2010, 546000 en 2015, soit un taux de croissance de $22 \%$ /an (MoHT, 2011 et 2016).

5. Traduction par nos soins.

6. Traduction par nos soins.

7. Traduction par nos soins.

\section{RÉSUMÉS}

Le lac Inlé, dans les collines de l'Est birman, présente un paysage très caractéristique de jardins flottants, créés au cours du $\mathrm{xx}^{\mathrm{e}}$ siècle par l'ethnie locale intha. Ces derniers ont réussi à mettre en place un système agricole très intensif, fortement connecté au reste de la Birmanie et du monde. 
Dans le contexte de l'ouverture de la Birmanie au monde, ce paysage extrêmement spécifique est valorisé par les acteurs touristiques. Cependant, ces derniers ont tendance à occulter la dimension intensive, mondialisée, innovante de cette pratique agricole. Au contraire, leurs discours présentent les jardins flottants comme des symboles de la «tradition», de l'« authenticité » de la « Birmanie intemporelle».

Inle Lake, in the hills of East Burma, presents the highly characteristic landscape of floating gardens which were created during the 20th century by the local ethnic group of the Intha. The Intha have succeeded in establishing a very intensive agricultural system with strong links with the rest of the country and the world. In the context of the opening up of Burma, this remarkable landscape is promoted by the professionals of the tourism sector, however, they tend to avoid showing the intensive, globalised and innovative approach this agricultural activity represents. On the contrary, they present the floating gardens as symbols of timeless Burmese "tradition" and "authenticity".

\section{INDEX}

Keywords : Burma/Myanmar, Inle Lake, agriculture, tourism, garden, globalisation

Mots-clés : Birmanie/Myanmar, lac Inlé, agriculture, tourisme, jardin, mondialisation

\section{AUTEUR}

\section{MARTIN MICHALON}

Il est doctorant en géographie du tourisme, Centre Asie du Sud-Est (CASE), École des hautes études en sciences sociales (EHESS).

martin.michalon[at]ehess[dot]fr et martin.michalon[at]yahoo[dot]fr 